\title{
Reststrahl band-assisted photocurrents in epitaxial graphene layers
}

\author{
P. Olbrich, ${ }^{1}$ C. Drexler, ${ }^{1}$ L. E. Golub, ${ }^{2}$ S. N. Danilov, ${ }^{1}$ V. A. Shalygin, ${ }^{3}$ R. Yakimova, ${ }^{4}$ S. Lara-Avila,,${ }^{5}$ S. Kubatkin, ${ }^{5}$ \\ B. Redlich, ${ }^{6}$ R. Huber, ${ }^{1}$ and S. D. Ganichev ${ }^{1}$ \\ ${ }^{1}$ Terahertz Center, University of Regensburg, 93040 Regensburg, Germany \\ ${ }^{2}$ Ioffe Physical-Technical Institute, Russian Academy of Sciences, 194021 St. Petersburg, Russia \\ ${ }^{3}$ St. Petersburg State Polytechnic University, 195251 St. Petersburg, Russia \\ ${ }^{4}$ Linköping University, S-58183 Linköping, Sweden \\ ${ }^{5}$ Chalmers University of Technology, S-41296 Göteborg, Sweden \\ ${ }^{6}$ Radboud University Nijmegen, Institute for Molecules and Materials, FELIX Facility, Toernooiveld 7 , \\ 6525 ED Nijmegen, The Netherlands
}

(Received 1 August 2013; published 17 December 2013)

\begin{abstract}
We report on the observation of the reststrahl band-assisted photocurrents in epitaxial graphene on SiC excited by infrared radiation. The peculiar spectral dependence for frequencies lying within the reststrahl band of the $\mathrm{SiC}$ substrate provides a direct and noninvasive way to probe the electric field magnitude at atomic distances from the material's surface. Furthermore our results reveal that nonlinear optical and optoelectronic phenomena in two-dimensional crystals and other atomic scale structures can be giantly enhanced by their deposition on a substrate with negative dielectric constant.
\end{abstract}

DOI: 10.1103/PhysRevB.88.245425

PACS number(s): 78.67.Wj, 42.70.Nq, 73.50.Pz, 81.05.ue

\section{INTRODUCTION}

Since the discovery of graphene, optical and optoelectronic properties of two-dimensional (2D) crystals have attracted continuously growing attention. ${ }^{1}$ A keen interest has been motivated by the prospective application of mono- or fewlayer systems in nonlinear optics, ${ }^{2-6}$ solar cells, ${ }^{7}$ displays,${ }^{8}$ optoelectronics, ${ }^{9}$ sensors, ${ }^{10,11}$ or plasmonic devices. ${ }^{12,13}$ Bridging the size mismatch between macroscopic photonics and atomic-scale integrated electronics all these concepts universally depend on a key quantity: the local optical fields acting on the charge carriers in 2D systems. These fields deviate from the emitted or incident waves due to the dielectric environment of the supporting substrate. A mesoscopic description has been invoked to obtain electromagnetic fields in the vicinity of a fictitious effective medium characterized by a mathematically sharp interface and bulk dielectric functions. ${ }^{12-15}$ Yet, in the extreme limit of an atomically thin system a mesoscopic model is not justified a priori and electromagnetic fields may be altered by the modified polarization response of the surface structure.

At the same time, the precise knowledge of the local electromagnetic fields is particularly important for phenomena that scale nonlinearly with the field amplitude. Examples range from optical nonlinearities to high-frequency transport as studied in 2D crystals, ${ }^{2-6,12-20}$ carbon nanotubes, ${ }^{21-24}$ topological insulators, ${ }^{25-27}$ and single molecules. ${ }^{28}$ Nonetheless, measuring electric fields on atomic distances is challenging. Surface-confined plasma oscillations in graphene have been shown to change their dispersion sensitively in the spectral vicinity of the reststrahl band ${ }^{29}$ of substrates. ${ }^{30}$ They could, thus, be basically used as sensors for the local dielectric environment. However, plasmon polaritons or phonon polaritons extend over comparably large distances into the substrate and into the air rather than being localized at a two-dimensional layer. Their properties depend on the entire dielectric environment over a layer of a thickness of tens to hundreds of nanometers towards either side of the graphene sample. ${ }^{31}$ Even for near-field microscopy reaching extreme subwavelength spatial resolution ${ }^{13,32}$ or a recent approach tracing the natural optical-frequency magnetic dipole transitions in lanthanide ions ${ }^{33}$ the atomic scale has been out of reach.

Here, we demonstrate that measurements of photoelectric effects in graphene deposited on a substrate provide a direct way to probe the electric field magnitude at atomic distances from the material's surface. We show that second-order photoelectric effects excited in graphene exhibit a peculiar spectral dependence for frequencies lying within the reststrahl band of the $\mathrm{SiC}$ substrate. The resonances of the photocurrents are attributed to the variation of the out-of-plane and in-plane components of the radiation electric field acting on electrons that are confined in the graphene layer deposited at a distance $d \approx 2 \AA$ from the $\mathrm{SiC}$ surface (see Fig. 1 and Ref. 34). Our analysis of the field distribution based on the macroscopic Fresnel formulas surprisingly well describes all experimental findings, while there remain quantitative discrepancies. As an important result, the observed reststrahl band-assisted photocurrent also clearly demonstrates that nonlinear optical and optoelectronic phenomena in 2D crystals can be giantly enhanced by a proper combination of the spectral range and substrate material.

\section{EXPERIMENTAL DETAILS AND SAMPLES PREPARATION}

Photocurrents have been observed in large area $n$ type graphene monolayer samples ${ }^{17,35}$ at room temperature. Figure 1(a) depicts the experimental geometry. Radiation was applied in the $(x z)$ plane at an angle of incidence $\theta$, varied between $-30^{\circ}$ and $+30^{\circ}$ to the layer normal, $z$. The signals generated in the unbiased devices were measured via an amplifier with $20 \mathrm{MHz}$ bandwidth and recorded with a storage oscilloscope. The photocurrents were induced applying midinfrared radiation of the frequency tunable free electron laser "FELIX" at FOM-Rijnhuizen in the Netherlands. ${ }^{36,37}$ The laser operated in the spectral range between 7 and $15 \mu \mathrm{m}$ 

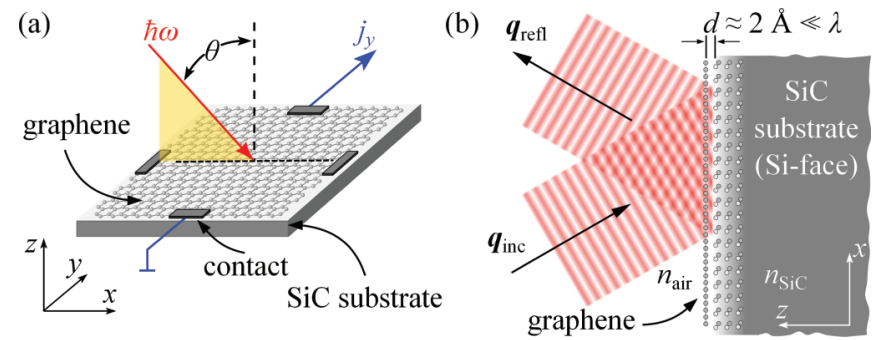

FIG. 1. (Color) (a) Experimental geometry. (b) Reflection of a plane wave from a graphene layer on a SiC substrate deposited at distances of about $2 \AA$ from the surface.

(corresponding to photon energies from $\hbar \omega \approx 180$ to $90 \mathrm{meV}$ ). The output pulses of light from FELIX were chosen to be $\approx 2 \mathrm{ps}$ long with peak power $P \approx 150 \mathrm{~kW}$, separated by $1 \mathrm{~ns}$, in a train (or "macropulse") of $5 \mu$ s duration. The beam has an almost Gaussian profile with a spot diameter of about $1 \mathrm{~mm}$, which is measured by a pyroelectric camera. ${ }^{38,39}$ The laser spot was always smaller than the sample size allowing us to avoid illumination of contacts or sample edges and, consequently, to study only photocurrents generated in pristine graphene. ${ }^{17}$ The radiation intensity $I$ and electric field $E_{0}$ on the sample during the micropulse were about $20 \mathrm{MW} / \mathrm{cm}^{2}$ and $120 \mathrm{kV} / \mathrm{cm}$, respectively. The macropulses had a repetition rate of $10 \mathrm{~Hz}$. The room temperature photoresponse was studied in the directions perpendicular and parallel to the light incidence plane. The results obtained applying the radiation of FELIX provide the full information about functional behavior of the photocurrent, however, the evaluation of the current magnitude in response to such short pulses is not straightforward. Thus, to calibrate the photocurrent response we additionally measured the current excited by the radiation of a line-tunable continuous wave $\mathrm{CO}_{2}$ laser with power $P$ of about $40 \mathrm{~mW}$. Though operating in a narrower spectral range (from 9.2 to $10.8 \mu \mathrm{m}$ ) it provides radiation in the vicinity of the upper limit of the reststrahl band and, therefore, is appropriated for the determination of the photocurrent value in the spectral region under study. The radiation power was controlled by a photon drag detector ${ }^{40}$ and/or a mercury cadmium telluride detector.

The experiments were carried out on several large area graphene monolayer samples epitaxially grown on the Si-terminated face of a $4 \mathrm{H}-\mathrm{SiC}(0001)$ semi-insulating substrate ${ }^{16,41}$ at $T=2000^{\circ} \mathrm{C}$ and $1 \mathrm{~atm}$ argon gas pressure..$^{42}$ The layers are $n$ doped due to the charge transfer from $\mathrm{SiC}$ with a measured electron concentration in the range of $1.5 \times 10^{12}$ to $7 \times 10^{12} \mathrm{~cm}^{-2}$, Fermi energy $E_{F}$ ranging from 160 to $300 \mathrm{meV}$, and mobility of about $10^{3} \mathrm{~cm}^{2} / \mathrm{V} \mathrm{s}$ at room temperature. ${ }^{16-18}$ The samples are characterized by a strong structure inversion asymmetry as demonstrated by the study of the magnetic quantum ratchet effect. ${ }^{35}$ Squares with dimensions of $5 \times 5 \mathrm{~mm}^{2}$ were patterned on graphene using standard electron beam (e-beam) lithography and oxygen plasma etching. Four metallic contacts on the periphery of graphene were produced by straightforward deposition of $\mathrm{Ti} / \mathrm{Au}(3 / 100 \mathrm{~nm})$ through a lithographically defined mask, followed by lift-off. Ohmic contacts have been prepared at the center of the edges, with a resistance of about $2 \mathrm{k} \Omega$ between opposed contacts.

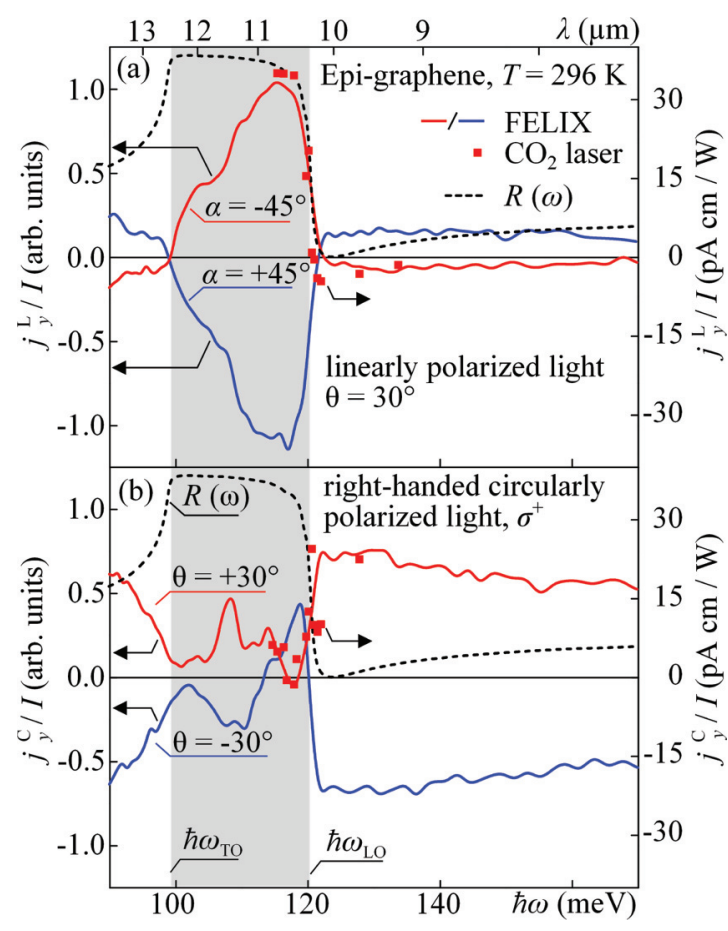

FIG. 2. (Color) Spectra of the photocurrent excited by (a) linearly and (b) circularly polarized radiation.

\section{EXPERIMENTAL RESULTS}

Illuminating an unbiased graphene layer with polarized radiation at oblique incidence we detected a photocurrent signal whose spectral behavior is shown in Fig. 2. Panel (a) shows the photocurrent $j_{y}^{L}$ excited by linearly polarized radiation with the angle between the polarization vector and the plane of incidence $\alpha= \pm 45^{\circ}$. A remarkable observation is that the current in the range of photon energies $\hbar \omega$ between about 99 and $120 \mathrm{meV}$ changes sign while its value increases by more than an order of magnitude compared to that excited by light with lower and higher frequencies. Despite the fact that the photocurrent shows a peculiar spectral dependence its overall functional behavior remains unchanged in the whole frequency range: The current (i) scales linearly with the radiation intensity $I \propto E_{0}^{2}$, (ii) is characterized by a short response time, and (iii) varies with the angle $\alpha$ and the angle of incidence $\theta$ as $j_{y}^{L}=L \sin 2 \alpha \sin \theta E_{0}^{2}$, where $L$ is a prefactor.

Besides the transversal photocurrent discussed in the paper, linearly polarized radiation also excites a current flowing along the light propagation direction, which varies as $j_{x}^{L}=$ $L \cos 2 \alpha \sin \theta E_{0}^{2}$ (not shown). The spectral dependence of the latter photocurrent is shown in Fig. 3 exhibiting resonancelike behavior similar to the transversal one. A helicitydependent photocurrent was not detected in longitudinal geometry. The overall functional behavior of the longitudinal photocurrent is in agreement with the phenomenological theory and microscopic picture of the photon drag and photogalvanic effects. ${ }^{16,18}$

Studying the radiation reflection reveals that the position and width of the photocurrent resonance matches well the reststrahl band of the $\mathrm{SiC},{ }^{30,43}$ which is characterized by an almost total reflection $R(\omega)$ between the longitudinal, $\hbar \omega_{\mathrm{LO}}$, 


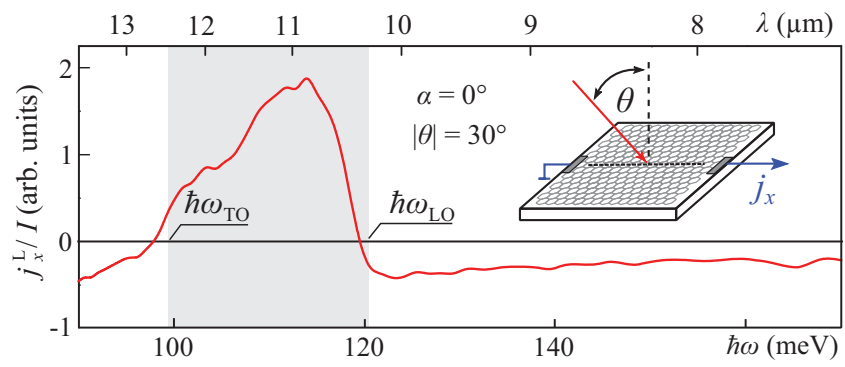

FIG. 3. (Color online) Spectra of the longitudinal photocurrent $j_{x}^{L}$ measured for $|\theta|=30^{\circ}$ and the azimuth angle $\alpha=0^{\circ}$. The data are obtained applying linearly polarized radiation of the free electron laser "FELIX." Gray area indicates the range of the reststrahl band of $\mathrm{SiC}$. The inset shows the experimental geometry.

and transversal, $\hbar \omega_{\mathrm{TO}}$, optical phonon energies. Figure 4 shows the reflection spectra of graphene and $\mathrm{SiC}$ samples. It is seen that the reflection spectra of graphene layer on $\mathrm{SiC}$ slightly deviates from that of the pure $\mathrm{SiC}$ substrate (see dotted line in Fig. 4). This result is in agreement with the data of Ref. 30 and is attributed to the substrate phonon-induced surface plasmonpolariton formation in epitaxial graphene.

The photocurrent is also observed for elliptically (circularly) polarized radiation, obtained via rotation of the Fresnel rhombus by the angle $\varphi$. By that we controllably vary the degree of linear, $P_{1}=(\sin 4 \varphi) / 2$, and circular, $P_{\mathrm{c}}=\sin 2 \varphi$, polarization, respectively. Figure 5(a) shows the polarization dependence of $j_{y}$ measured in the vicinity of $\hbar \omega_{\mathrm{LO}}$. The detected photocurrent can be well fitted by $j_{y}=j_{y}^{L}+j_{y}^{C}=$ $[(L / 2) \sin 4 \varphi+C \sin 2 \varphi] \sin \theta E_{0}^{2}$ as shown in Figs. 5(b) and 5 (c) for photon energies below and above $\hbar \omega_{\mathrm{LO}}$, respectively. Here the linear photocurrent $j_{y}^{L}$, given by the fourth harmonics of $\varphi$, is just the above discussed current in response to linearly polarized radiation but excited by elliptically polarized light. The circular current $j_{y}^{C}$ stems from the radiation helicity and is proportional to $P_{\mathrm{c}}$. Figure 5 reveals the crossover from the dominating circular $(\propto \sin 2 \varphi)$ to linear $(\propto \sin 4 \varphi)$ photocurrent contributions in the vicinity of $\hbar \omega_{\text {LO }}$. The spectral behavior of the circular contribution $j^{C}$ is shown for righthanded circular polarization in Fig. 2(b). Similar to the linear photocurrent, we found here a peculiar spectral behavior within the reststrahl band. However, by contrast to $j_{y}^{L}$, the circular

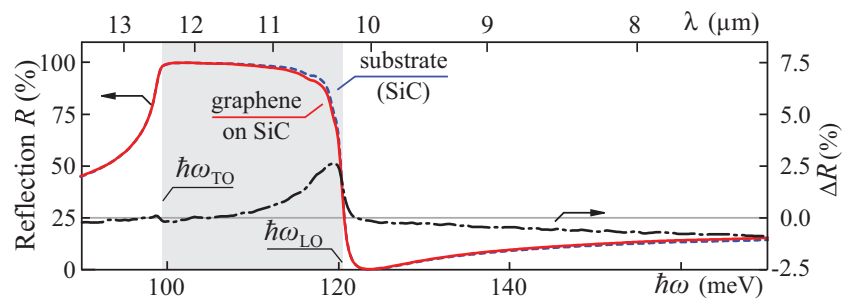

FIG. 4. (Color online) Dashed and solid lines show the reflection spectra of graphene on $\mathrm{SiC}$ substrate and pure $\mathrm{SiC}$, respectively. Dotted line shows the difference between the two curves. The spectra are measured with a Fourier spectrometer. Gray area shows the range of the reststrahl band of $\mathrm{SiC}$ limited by the energies of transverse and longitudinal optical phonons.

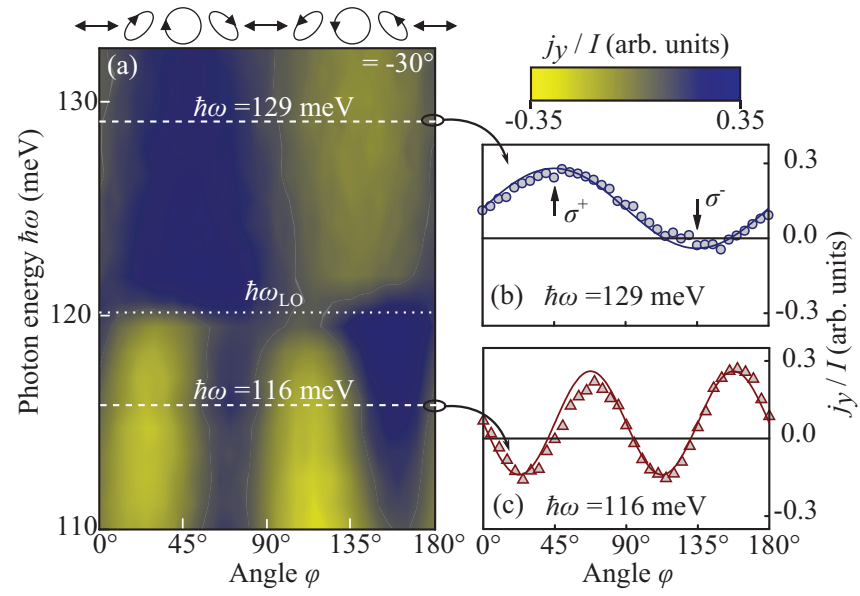

FIG. 5. (Color) (a) Photocurrent $j_{y} / I$ as a function of $\hbar \omega$ and angle $\varphi$ (polarization states are sketched above). (b) and (c) show corresponding dependencies obtained for fixed $\hbar \omega$.

photocurrent is not enhanced but rather suppressed. It has a complex spectral behavior exhibiting a change of sign and a peak close to the center of the reststrahl band.

\section{DISCUSSION}

The microscopic origin of the photocurrent outside of the reststrahl band has been previously investigated in Ref. 18 . It has been demonstrated that the current is caused by a sum of the photon drag (PDE) and photogalvanic (PGE) effects of comparable strength. In particular, it has been shown that the photon drag effect in graphene is caused by a simultaneous action of the electric and magnetic field components of the infrared radiation and, in fact, can be classified as a dynamic Hall effect. ${ }^{16,18}$ The origin of the PGE is the asymmetry of electron scattering induced by radiation and structure inversion asymmetry. ${ }^{18,44,45}$ The addressed above fact that all characteristic features of the photocurrent are the same within and outside the reststrahl band, indicates that its microscopic origin remains unchanged. Actually, this is not surprising because for $\hbar \omega \ll E_{\mathrm{F}}$ and room temperature no resonances are expected for the light-matter interaction in pristine graphene. However, one can expect dramatic modifications of local electric fields acting on carriers in graphene for frequencies within the reststrahl band of the substrate, which is characterized by a negative dielectric constant of the material. Indeed the coincidence of the increased reflection with the observed resonance of the photocurrent clearly indicates the common origin of both effects.

As we show below, the resonant photoresponse can be well understood considering only the spectral behavior of the inplane and out-of-plane radiation electric field components, without going into microscopic details. The required electric field components responsible for the photocurrent formation provide the phenomenological theory of PDE and PGE. In line with the experiment we consider the transverse photocurrent $j_{y}$ generated in the direction perpendicular to the incidence plane $(x z)$. Following Ref. 6 the current density due to the 
photon drag effect is given by

$$
j_{y}^{\mathrm{PDE}}=T q_{x}\left(E_{x} E_{y}^{*}+E_{x}^{*} E_{y}\right)+T^{\prime} q_{x} i\left(E_{y} E_{x}^{*}-E_{x} E_{y}^{*}\right),
$$

and due to the photogalvanic effect by

$$
j_{y}^{\mathrm{PGE}}=\chi\left(E_{z} E_{y}^{*}+E_{y} E_{z}{ }^{*}\right)+\gamma i\left(E_{z} E_{y}^{*}-E_{y} E_{z}{ }^{*}\right) .
$$

Here $E$ is the electric field acting on electrons, and $T$ and $\chi$ are coefficients describing, respectively, the linear PDE and PGE. These currents are proportional to the linear polarization degree $P_{l}$ given by symmetrical combinations of electric field components. The two remaining coefficients correspond to the circular PDE $\left(T^{\prime}\right)$ and PGE $(\gamma)$ currents, which reverse the direction upon switching the photon helicity given by $i\left(\boldsymbol{E} \times \boldsymbol{E}^{*}\right) /|E|^{2} \cdot \boldsymbol{q} / q$.

In the following analysis we assume coefficients $\chi, \gamma$, $T$, and $T^{\prime}$ to be frequency independent in the studied narrow frequency range and focus on the frequency variation of the electric field components only. This assumption is reasonable for the considered experimental conditions because for room temperature, $\hbar \omega \ll E_{\mathrm{F}}$, and $\omega \tau \sim 1$, the radiation absorption is caused by Drude-like indirect intraband optical transitions, and hence, $\chi, \gamma, T$, and $T^{\prime}$ have smooth frequency dependencies. ${ }^{16,18}$ Moreover, we disregard a possible influence of graphene itself on the electric field magnitudes.

To obtain the frequency dependence of the required electric field components we use macroscopic Fresnel formulas, which, strictly speaking, are applicable for representation of dielectric medium by a homogeneous function $\varepsilon(\omega)$, which is independent of the position within the medium. In this approach the electric fields are formed by superposition of the incident and reflected waves [see Fig. 1(b)]. The electric fields are described by the Fresnel transmission coefficients, which reflect the spectral behavior of the dielectric function of the substrate, $\varepsilon(\omega)$. These coefficients are for oblique incident radiation given by

$$
\begin{aligned}
t_{s} & =\frac{2 \cos \theta_{0}}{\sqrt{\varepsilon-\sin ^{2} \theta_{0}}+\cos \theta_{0}}, \\
t_{p} & =\frac{2 \sqrt{\varepsilon} \cos \theta_{0}}{\sqrt{\varepsilon-\sin ^{2} \theta_{0}}+\varepsilon \cos \theta_{0}} .
\end{aligned}
$$

At an oblique incidence of radiation on the dielectric media with $\varepsilon(\omega)$ the wave-vector component in the surface plane $q_{x}=(\omega / c) \sin \theta_{0}$ is continuous while the normal wave-vector component inside the medium $q_{z}^{\text {in }}=(\omega / c)(n+i \varkappa)$, where ${ }^{46}$

$$
\begin{aligned}
& n=\sqrt{\frac{\sqrt{\left(\varepsilon^{\prime}-\sin ^{2} \theta_{0}\right)^{2}+\varepsilon^{\prime \prime 2}}+\varepsilon^{\prime}-\sin ^{2} \theta_{0}}{2},} \\
& \varkappa=\sqrt{\frac{\sqrt{\left(\varepsilon^{\prime}-\sin ^{2} \theta_{0}\right)^{2}+\varepsilon^{\prime \prime 2}}-\left(\varepsilon^{\prime}-\sin ^{2} \theta_{0}\right)}{2} .}
\end{aligned}
$$

Here $\varepsilon^{\prime}$ and $\varepsilon^{\prime \prime}$ are the real and imaginary parts of the dielectric function (7), respectively.

As is well known, the dielectric function of the substrate $\varepsilon(\omega)$ exhibits a strong anomaly within the reststrahl band ${ }^{29}$

$$
\varepsilon(\omega)=\varepsilon_{\infty}+\frac{\varepsilon_{0}-\varepsilon_{\infty}}{1-\left(\omega / \omega_{\mathrm{TO}}\right)^{2}-i \omega \Gamma / \omega_{\mathrm{TO}}^{2}},
$$

where $\Gamma$ is the damping constant, and $\varepsilon_{0}$ and $\varepsilon_{\infty}$ are the low- and high-frequency dielectric constants, respectively. The complex dielectric function and, consequently, the complex refractive index $\sqrt{\varepsilon}=n+i \varkappa$, determine the frequency dependence of the electric field components. Here $n$ is refractive index and $\varkappa$ the extinction coefficient. The in-plane components $E_{x}$ and $E_{y}$ are continuous and found from the Maxwell equation $\operatorname{div} \boldsymbol{E}=0$ yielding

$$
E_{x}=t_{p} E_{0 p}(n+i \varkappa) \Xi, \quad E_{y}=t_{s} E_{0 s},
$$

where $E_{0 s}, E_{0 p}$ are the corresponding parts of the incident wave amplitude, $\Xi=1 / \sqrt{n^{2}+\varkappa^{2}+\sin ^{2} \theta_{0}}$, and $t_{s}, t_{p}$ are the standard Fresnel amplitude transmission coefficients for $s$ and $p$ polarizations.

While the in-plane field components are continuous at the air/SiC interface, its normal component $E_{z}$ is discontinuous having different values inside and outside $\mathrm{SiC}$ :

$$
E_{z}^{\text {in }}=-t_{p} E_{0 p} \sin \theta_{0} \Xi, \quad E_{z}^{\text {out }}=\varepsilon E_{z}^{\text {in }} .
$$

Using these solutions we obtain spectral behavior of the PDE and PGE, respectively. We start with the PDE current given by Eq. (1), the in-plane components $E_{x, y}$, and photon wave vector $q_{x}=(\omega / c) \sin \theta_{0}$. All these quantities are continuous, therefore, we obtain one solution for each, linear and circular, photocurrents:

$$
\begin{aligned}
j_{y}^{L} & =\frac{\omega}{c} \sin \theta_{0} P_{1}\left|E_{0}\right|^{2} \\
& \times\left[\left(n T+\varkappa T^{\prime}\right) \operatorname{Re}\left(t_{p}^{*} t_{s}\right)+\left(\varkappa T-n T^{\prime}\right) \operatorname{Im}\left(t_{p}^{*} t_{s}\right)\right] \Xi,
\end{aligned}
$$

$$
\begin{aligned}
j_{y}^{C}= & \frac{\omega}{c} \sin \theta_{0} P_{\mathrm{c}}\left|E_{0}\right|^{2} \\
& \times\left[\left(\varkappa T-n T^{\prime}\right) \operatorname{Re}\left(t_{p}^{*} t_{s}\right)-\left(n T+\varkappa T^{\prime}\right) \operatorname{Im}\left(t_{p}^{*} t_{s}\right)\right] \Xi .
\end{aligned}
$$

Here the linear polarization degree is introduced according to $P_{l}=\left(E_{0 p} E_{0 s}^{*}+E_{0 s} E_{0 p}^{*}\right) /\left|E_{0}\right|^{2}$, and the circular polarization degree is given by $P_{c}=i\left(E_{0 p} E_{0 s}^{*}-E_{0 s} E_{0 p}^{*}\right) /\left|E_{0}\right|^{2}$. The above equations yield at first glance a surprising result. By contrast to Eq. (1) the obtained linear and circular PDE currents are determined by both $T$ and $T^{\prime}$ coefficients. This comes from the fact that, within the reststrahl band, the radiation acting on the electrons in the graphene layer becomes elliptically polarized even for irradiation with purely linear or circular light. $^{47}$

Figure 6(b) shows the calculated spectra of the linear and circular photon drag effect. Calculations are carried out for the dominating contribution of the circular PDE - the fact which clearly follows from the data outside the reststrahl band. Within the reststrahl band the situation changes. Due to the polarization transformation addressed above, the $T^{\prime}$ contribution gives rise to the enhancement of the linear PDE. At the same time the decrease of the radiation helicity results in suppression of the circular photocurrent. While our calculations of the photon drag effect confirm the enhancement/suppression of the linear/circular photocurrent within the reststrahl band they do not describe the double sign inversion and significant value of the linear photocurrent detected outside the reststrahl band [see Fig. 2(a)]. 
(a)
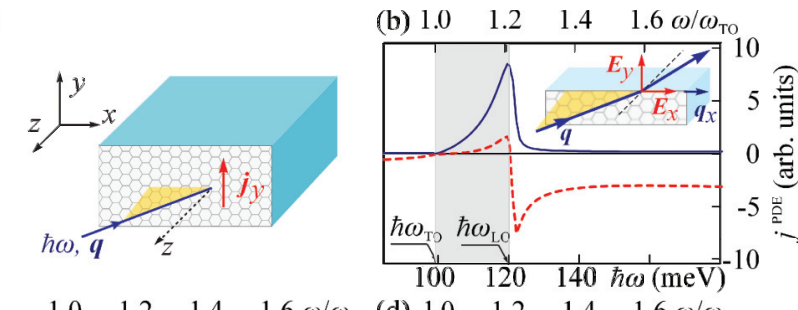

(c) $\begin{array}{rrrrrrr}1.0 & 1.2 & 1.4 & 1.6 \omega / \omega_{\text {то }} \text { (d) } 1.0 & 1.2 & 1.4 & 1.6 \omega / \omega_{\text {Tо }}\end{array}$

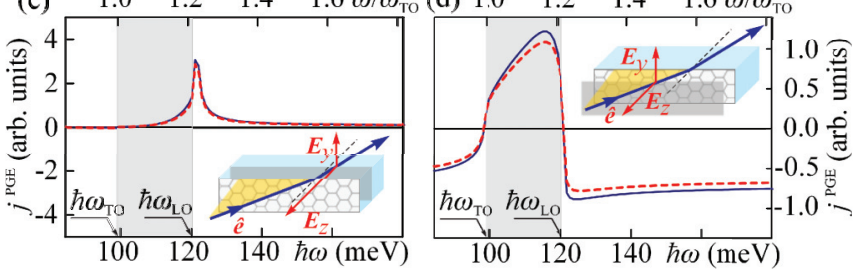

FIG. 6. (Color) (a) Experimental geometry. (b)-(d) Calculated spectra of the linear (solid) and circular (dashed) photocurrents. (b) Photon drag effect. (c) and (d) Photogalvanic effect caused by the electric field in the SiC side, $\boldsymbol{E}^{\text {in }}$, and in the air side of the air/SiC interface, $\boldsymbol{E}^{\text {out }}$, respectively. The calculations are performed for $\theta_{0}=30^{\circ}, \gamma / \chi=0.9$, and $T^{\prime} / T=18$, as well as obtained from the reflection data $\Gamma=0.01 \omega_{\mathrm{TO}}, \varepsilon_{\infty}=6.52$, and $\varepsilon_{0}=9.66$ for $\mathrm{SiC}$ (see also Ref. 43). The insets shows the electric field components considered in the calculations.

To obtain a better agreement we consider the photogalvanic effect which, according to Ref. 18, should yield a comparable contribution to the total photocurrent excited by infrared radiation. It follows from Eq. (2) that all PGE contributions require a normal component of the electric field, which is discontinuous at the interface. Therefore, from Eqs. (2) and (9) we obtain different solutions for the photocurrents induced by the field in the air side of the air/SiC interface, $E_{z}^{\text {out: }}$

$$
\begin{aligned}
& j_{y}^{L}=-\sin \theta_{0} P_{1}\left|E_{0}\right|^{2}\left[\chi \operatorname{Re}\left(t_{p}^{*} t_{s}\right)+\gamma \operatorname{Im}\left(t_{p}^{*} t_{s}\right)\right] \Xi, \\
& j_{y}^{C}=-\sin \theta_{0} P_{\mathrm{c}}\left|E_{0}\right|^{2}\left[\gamma \operatorname{Re}\left(t_{p}^{*} t_{s}\right)+\chi \operatorname{Im}\left(t_{p}^{*} t_{s}\right)\right] \Xi,
\end{aligned}
$$

and from the field in the $\mathrm{SiC}$ side of the interface, $E_{z}^{\mathrm{in}}$ :

$$
\begin{aligned}
& j_{y}^{L}=-\sin \theta_{0} P_{1}\left|E_{0}\right|^{2}\left[\chi \operatorname{Re}\left(t_{p}^{*} \varepsilon^{*} t_{s}\right)+\gamma \operatorname{Im}\left(t_{p}^{*} \varepsilon^{*} t_{s}\right)\right] \Xi, \\
& j_{y}^{C}=-\sin \theta_{0} P_{c}\left|E_{0}\right|^{2}\left[\gamma \operatorname{Re}\left(t_{p}^{*} \varepsilon^{*} t_{s}\right)+\chi \operatorname{Im}\left(t_{p}^{*} \varepsilon^{*} t_{s}\right)\right] \Xi .
\end{aligned}
$$

The resulting calculations, applying the same parameters as that used for the PDE, are shown in Figs. 6(c) and 6(d). It is seen that both linear and circular PGE show a strong enhancement in the reststrahl band. The solutions for the electric field in the substrate [see Fig. 6(c)] yield a sharp peak close to $\hbar \omega_{\text {LO }}$ only and, thus, do not describe the complex spectral behavior of the photocurrent. Moreover, we did not detect any sharp peak at LO frequency, which supports the conclusion that this contribution to the total photocurrent is negligible. Good agreement with the experiment is obtained for the linear PGE excited by the electric field in the air side of the air/SiC interface [see Fig. 6(d)]. However, this solution does not describe the suppression of the circular photocurrent.
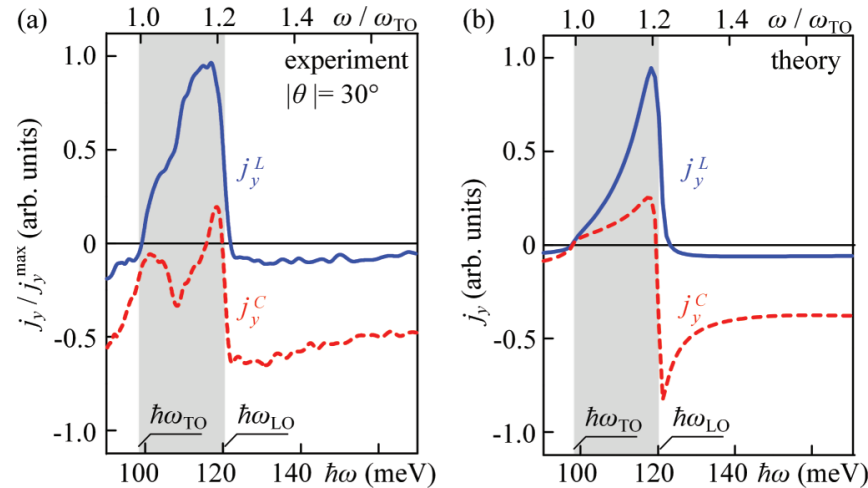

FIG. 7. (Color online) Spectral behavior of the linear (solid lines) and circular (dashed lines) photocurrents. (a) Experimental results. (b) Calculations of the total photocurrent considering electric field components $E_{x}, E_{y}$ and electric field component in the air side of the air/SiC interface $E_{z}^{\text {out }}$. Here we used a ratio of circular PDE to circular PGE equal to $\left(\omega_{T o} / c\right) T^{\prime} / \chi=0.3$ and the parameters given in the caption of Fig. 6.

We emphasize that this disagreement cannot be avoided simply by variation of the coefficients $\gamma$ and $\chi$.

Our calculations show that overall reasonable agreement can only be achieved by considering a superposition of both PDE and PGE photocurrents and taking into account the $z$ component of the electric field in the air side of the air/SiC interface. The corresponding results together with experimental data are shown for transversal photocurrent in Fig. 7. In fact, the calculations reflect all main features of the measured photocurrent, namely, (i) both circular and linear currents depend weakly on the frequency outside the reststrahl band; (ii) the linear current changes its sign and has a broad peak within the whole reststrahl band; (iii) the circular current is suppressed and has a peak at $\hbar \omega \approx 115 \mathrm{meV}$; and (iv) the linear current dominates the circular one within the reststrahl band and vice versa in the outside. We emphasize that a satisfying agreement is obtained despite the fact that the calculations are carried out for the homogeneous functions $\varepsilon(\omega)$, abrupt interfaces, and the disregarded influence of graphene on the radiation field. These simplifications used in our model or possible $E_{z}$ electric field induced charge transfer between graphene and $\mathrm{SiC}^{48}$ may be responsible for remaining small discrepancies. A comparison of the linear PDE and PGE components reveals that within the resonance the photon drag effect strongly dominates in the total current, whereas outside the resonance the main contribution comes from the linear PGE. As for the circular photocurrent the PDE is responsible for the photoresponse in the whole studied spectral range.

In the above analysis the magnitudes and spectral behavior of particular electric field components have been calculated after Eqs. (8) and (9) using the set of parameters given in the caption to Fig. 6. The complex spectral behavior of the dielectric constant epsilon within the reststrahl band [see Eq. (7)] results in the enhancement of particular electric field components. As an example the $E_{x} E_{y}$ product changes by a factor of 8.4 upon variation of radiation frequency from $\omega$ lying outside the reststrahl band $\left(\omega=0.5 \omega_{\mathrm{TO}}\right)$ to that within the restrahl band $\left(\omega=1.2 \omega_{\mathrm{TO}}\right)$ where the maximum of this 
product is reached. While it is tempting to assume, a priori, that the electric field in the vicinity of a surface is "simply" determined by the Fresnel formulas, this approach, after careful consideration, is not trivial. In fact, the derivation of the Fresnel formulas assumes interfaces of fictitious homogeneous dielectric media described by dielectric functions. In the field of optics this assumption is usually valid since the wavelengths of light as well as penetration depths of evanescent waves exceed interatomic distances by several orders of magnitude. On an atomic length scale, however, the physics could be quite different at surfaces, because the local polarizability of the single atomic layer may deviate from the bulk values.

\section{SUMMARY}

To conclude, our results demonstrate that photocurrents in graphene deposited on a medium with a negative dielectric constant can be efficiently used for studies of how an electric field acts on the atomic scale. While the described approach is limited to the spectral range defined by the reststrahl band of the substrate, radiation of any desired frequency can be analyzed using negative $\varepsilon$ of artificially made periodic structures, such as metamaterials. ${ }^{49}$ As an important result, our findings demonstrate that optical and optoelectronic phenomena can be giantly enhanced in strictly 2D systems and other nanoscale systems if these structures are deposited on a substrate with a negative $\varepsilon$. This result is of importance for various kinds of applications, in particular, those making use of effects proportional to higher orders of the electric field.

\section{ACKNOWLEDGMENTS}

We thank S. A. Tarasenko and M. M. Voronov for helpful discussions. Support from DFG (SPP 1459 and GRK 1570), Linkage Grant of IB of BMBF at DLR, RFBR, and POLAPHEN is acknowledged. We gratefully acknowledge the support by the Stichting voor Fundamenteel Onderzoek der Materie (FOM) in providing the required beam time on FELIX.
${ }^{1}$ K. S. Novoselov, V. I. Fal'ko, L. Colombo, P. R. Gellert, M. G. Schwab, and K. Kim, Nature (London) 490, 192 (2012).

${ }^{2}$ J. J. Dean and H. M. van Driel, Appl. Phys. Lett. 95, 261910 (2009).

${ }^{3}$ E. Hendry, P. J. Hale, J. Moger, A. K. Savchenko, and S. A. Mikhailov, Phys. Rev. Lett. 105, 097401 (2010).

${ }^{4}$ M. Dragoman, D. Neculoiu, G. Deligeorgis, G. Konstantinidis, D. Dragoman, A. Cismaru, A. A. Muller, and R. Plana, Appl. Phys. Lett. 97, 093101 (2010).

${ }^{5}$ G. R. Hotopan, S. Ver-Hoeye, C. Vazquez-Antuna, R. CamblorDiaz, M. Fernandez-Garcia, F. Las Heras Andres, P. Alvarez, and R. Menéndez, Prog. Electromagn. Res. 118, 57 (2011).

${ }^{6}$ M. M. Glazov and S. D. Ganichev, Phys. Reports, doi:10.1016/j.physrep.2013.10.003.

${ }^{7}$ K. J. Tielrooij, J. C. W. Song, S. A. Jensen, A. Centeno, A. Pesquera, A. Zurutuza Elorza, M. Bonn, L. S. Levitov, and F. H. L. Koppens, Nat. Phys. 9, 248 (2013).

${ }^{8}$ S. Bae et al., Nat. Nanotechnol. 5, 574 (2010).

${ }^{9}$ T. Gu, N. Petrone, J. F. McMillan, A. van der Zande, M. Yu, G. Q. Lo, D. L. Kwong, J. Hone, and C. W. Wong, Nat. Photonics 6, 554 (2012).

${ }^{10}$ L. Vicarelli, M. S. Vitiello, D. Coquillat, A. Lombardo, A. C. Ferrari, W. Knap, M. Polini, V. Pellegrini, and A. Tredicucci, Nat. Mater. 11, 865 (2012).

${ }^{11}$ N. L. Rangel, A. Gimenez, A. Sinitskii, and J. M. Seminario, J. Phys. Chem. C 115, 12128 (2011).

${ }^{12}$ J. Chen et al., Nature (London) 487, 77 (2012).

${ }^{13}$ Z. Fei et al., Nature (London) 487, 82 (2012).

${ }^{14}$ M. Jablan, H. Buljan, and M. Soljacic, Phys. Rev. B 80, 245435 (2009).

${ }^{15}$ F. H. L. Koppens, D. E. Chang, and F. J. G. de Abajo, Nano Lett. 11, 3370 (2011).

${ }^{16}$ J. Karch et al., Phys. Rev. Lett. 105, 227402 (2010).

${ }^{17}$ J. Karch et al., Phys. Rev. Lett. 107, 276601 (2011).

${ }^{18}$ C. Jiang, V. A. Shalygin, V. Y. Panevin, S. N. Danilov, M. M. Glazov, R. Yakimova, S. Lara-Avila, S. Kubatkin, and S. D. Ganichev, Phys. Rev. B 84, 125429 (2011).
${ }^{19}$ D. Sun, J. Rioux, J. E. Sipe, Y. Zou, M. T. Mihnev, C. Berger, W. A. de Heer, P. N. First, and T. B. Norris, Phys. Rev. B 85, 165427 (2012).

${ }^{20}$ L. Prechtel, L. Song, D. Schuh, P. Ajayan, W. Wegscheider, and A. W. Holleitner, Nat. Commun. 3, 646 (2012).

${ }^{21}$ Carbon Nanotubes: Advanced Topics in the Synthesis, Structure, Properties and Applications, edited by A. Jorio, G. Dresselhaus, and M. S. Dresselhaus (Springer, New York, 2008).

${ }^{22}$ E. L. Ivchenko and B. Spivak, Phys. Rev. B 66, 155404 (2002).

${ }^{23}$ G. Y. Slepyan, S. A. Maksimenko, V. P. Kalosha, A. V. Gusakov, and J. Herrmann, Phys. Rev. A 63, 053808 (2001).

${ }^{24}$ G. M. Mikheev, A. G. Nasibulin, R. G. Zonov, A. Kaskela, and E. I. Kauppinen, Nano Lett. 12, 77 (2012).

${ }^{25}$ X.-L. Qi, S. C. Zhang, and X. L. Qi, Rev. Mod. Phys. 83, 1057 (2011).

${ }^{26}$ P. Hosur, Phys. Rev. B 83, 035309 (2011).

${ }^{27}$ J. W. McIver, D. Hsieh, H. Steinberg, P. Jarillo-Herrero, and N. Gedik, Nat. Nanotechnol. 7, 96 (2012).

${ }^{28}$ J. Lupton, Adv. Mater. 22, 1689 (2010).

${ }^{29} \mathrm{P}$. Y. Yu and M. Cardona, Fundamentals of Semiconductors: Physics and Materials Properties (Springer, New York, 2010).

${ }^{30}$ B. K. Daas, K. M. Daniels, T. S. Sudarshan, and M. V. S. Chandrashekhar, J. Appl. Phys. 110, 113114 (2011).

${ }^{31}$ H. Yan, T. Low, W. Zhu, Y. Wu, M. Freitag, X. Li, F. Guinea, P. Avouris, and F. Xia, Nat. Photonics 7, 394 (2013).

${ }^{32}$ Z. Fei et al., Nano Lett. 11, 4701 (2011).

${ }^{33}$ T. H. Taminiau, S. Karaveli, N. F. van Hulst, and R. Zia, Nat. Commun. 3, 979 (2012).

${ }^{34}$ J. Borysiuk, R. Bożek, W. Strupiński, A. Wysmołek, K. Grodecki, R. Stępniewski, and J. M. Baranowski, J. Appl. Phys. 105, 023503 (2009).

${ }^{35}$ C. Drexler et al., Nat. Nanotechnol. 8, 104 (2013).

${ }^{36}$ G. M. H. Knippels, X. Yan, A. M. MacLeod, W. A. Gillespie, M. Yasumoto, D. Oepts, and A. F. G. van der Meer, Phys. Rev. Lett. 83, 1578 (1999). 
${ }^{37}$ W. Weber et al., Phys. Rev. B 77, 245304 (2008).

${ }^{38}$ E. Ziemann, S. D. Ganichev, W. Prettl, I. N. Yassievich, and V. I. Perel, J. Appl. Phys. 87, 3843 (2000).

${ }^{39}$ S. D. Ganichev, I. N. Yassievich, W. Prettl, J. Diener, B. K. Meyer, and K. W. Benz, Phys. Rev. Lett. 75, 1590 (1995).

${ }^{40}$ S. D. Ganichev, Ya. V. Terent'ev, and I. D. Yaroshetskii, Pis'ma Zh. Tekh. Fiz. 11, 46 (1985) [Sov. Tech. Phys. Lett. 11, 20 (1985)].

${ }^{41}$ A. Tzalenchuk, S. Lara-Avila, A. Kalaboukhov, S. Paolillo, M. Syväjärvi, R. Yakimova, O. Kazakova, T. J. B. M. Janssen, V. Fal'ko, and S. Kubatkin, Nat. Nanotechnol. 5, 186 (2010).

${ }^{42}$ K. V. Emtsev et al., Nat. Mater. 8, 203 (2009).

${ }^{43}$ L. Patrick and W. J. Chouke, Phys. Rev. B 2, 2255 (1970).

${ }^{44}$ S. D. Ganichev, E. L. Ivchenko, and W. Prettl, Physica E 14, 166 (2002).
${ }^{45}$ Note that the PGE current, being forbidden by symmetry in pristine graphene, is allowed in graphene layer on a substrate due to broken $z \rightarrow-z$ symmetry. The samples under study are characterized by a strong structure inversion asymmetry as demonstrated in Ref. 35.

${ }^{46}$ L. D. Landau and E. M. Lifshits, Course of Theoretical Physics, Electrodynamics of Continuous Media Vol. 8 (Pergamon, Oxford, 1984).

${ }^{47}$ The polarization transformation is caused by the existence of the imaginary parts of transmission coefficients $t_{p}$ and $t_{s}$ within the reststrahl band.

${ }^{48}$ S. Kopylov, A. Tzalenchuk, S. Kubatkin, and V. I. Falko, Appl. Phys. Lett. 97, 112109 (2010).

${ }^{49}$ L. Solymar and E. Shamonina, Waves in Metamaterials (Oxford University Press, New York, 2009). 